\title{
Aerodynamics and flight mechanics of MAV based on Coanda effect
}

\begin{abstract}
With the progress and development of Coandă Micro Air Vehicle (MAV) to date, various configurations have been proposed and developed. For the purpose of designing a Micro Air Vehicle (MAV), that could meet the desired mission and design requirements and can operate effectively in various environmental conditions, a workable theoretical analysis is to be developed. With such motivation, capitalizing on the basic fundamental principles, the aerodynamic forces acting on Coandă MAV configurations are revisited and analysed on the bases of the Fluid Dynamics and Flight Mechanics of a semi-spherical Coandă MAV configuration. The major objective of this paper is to analyse the forces and moments acting on the Coandă MAV as related to the geometry, flow, and motion parameters, using mathematical modelling. The mathematical model and derived performance measures in hover and translatory motion are shown to be capable in describing the physical phenomena of the flow field of the semi-spherical Coandă MAV. Results obtained are assessed and considered viable for preliminary design of such air vehicles and further elaborated development, including more flight dynamic manoeuvres.
\end{abstract}

Keyword: Circulation control; Coandă effect; Coandă MAV flight mechanics; Coandă MAV fluid dynamics; Micro Air Vehicle (MAV); Unmanned Aerial Vehicle (UAV) 